\title{
Block LDA for Face Recognition
}

\author{
Vo Dinh Minh Nhat, and Sungyoung Lee \\ Kyung Hee University - South of Korea \\ \{vdmnhat, sylee\}@oslab.khu.ac.kr
}

\begin{abstract}
Linear Discriminant Analysis (LDA) technique is an important and well-developed area of image recognition and to date many linear discrimination methods have been put forward. Despite these efforts, there persist in the traditional LDA some weaknesses. In this paper, we propose a new LDA-based method called Block LDA (BLDA) that can outperform the traditional Linear Dicriminant Analysis (LDA) methods. As opposed to conventional LDA, BLDA is based on 2D matrices rather than $1 \mathrm{D}$ vectors. That is, we firstly divides the original image into blocks. Then, we transform the image into a vector of blocks. By using row vector to represent each block, we can get the new matrix which is the representation of the image. Finally LDA can be applied directly on these matrices. In contrast to the between-class and within-class covariance matrices of LDA, the size of the these covariance matrices using BLDA is much smaller. As a result, BLDA has three important advantages over LDA. First, it is easier to evaluate the between-class and within-class covariance matrices accurately. Second, less time is required to determine the corresponding eigenvectors. And finally, block size could be changed to get the best results. Experiment results show our method achieves better performance in comparison with the other methods.
\end{abstract}

Index Terms - Linear Discriminant Analysis (LDA), Block Linear Discriminant Analysis (BLDA), face recognition.

\section{Introduction}

Face recognition research has been started in the late 70s and is one of the active and exciting researches in computer science and information technology areas since 1990 [1]. Generally, there are three phases for face recognition, mainly face representation, face detection, and face identification. Face representation is the first task, that is, how to model a face. The way to represent a face determines the successive algorithms of detection and identification. There are a variety of approaches for face representation, which can be roughly classified into three categories: template-based, feature-based, and appearance-based. The simplest template-matching approaches represent a whole face using a single template, i.e., a 2-D array of intensity, which is usually an edge map of the original face image. In a more complex way of template-matching, multiple templates may be used for each face to account for recognition from different 


\section{Vo Dinh Minh Nhat, and Sungyoung Lee}

viewpoints. Another important variation is to employ a set of smaller facial feature templates that correspond to eyes, nose, and mouth, for a single viewpoint. The most attractive advantage of template-matching is the simplicity, however, it suffers from large memory requirement and inefficient matching. In feature-based approaches, geometric features, such as position and width of eyes, nose, and mouth, eyebrow's thickness and arches, face breadth, or invariant moments, are extracted to represent a face. Feature-based approaches have smaller memory requirement and a higher recognition speed than template-based ones do. They are particularly useful for face scale normalization and 3D head model-based pose estimation. However, perfect extraction of features is shown to be difficult in implementation. Eigenfaces approach is one of the earliest appearance-based face recognition methods, which was developed by M. Turk and A. Pentland [2] in 1991. This method utilizes the idea of the PCA and decomposes face images into a small set of characteristic feature images called eigenfaces. Recognition is performed by projecting a new face onto a low dimensional linear "face space" defined by the eigenfaces, followed by computing the distance between the resultant position in the face space and those of known face classes.

The Fisherface method [4] combines PCA and the Fisher criterion [9] to extract the information that discriminates between the classes of a sample set. It is a most representative method of LDA. Nevertheless, Martinez et al. demonstrated that when the training data set is small, the Eigenface method outperforms the Fisherface method [7]. Should the latter be outperformed by the former? This provoked a variety of explanations. Liu et al. thought that it might have been because the Fisherface method uses all the principal components, but the components with the small eigenvalues correspond to high-frequency components and usually encode noise [11], leading to recognition results that are less than ideal. In line with this theory, they presented two enhanced Fisher linear discrimination (FLD) models (EFMs) [11] and an enhanced Fisher classifier [12] for face recognition. Their experiential explanation lacks sufficient theoretical demonstration, however, and EFM does not provide an automatic strategy for selecting the components. Chen et al. proved that the null space of the within-class scatter matrix contains the most discriminative information when a small sample size problem takes place [13]. Their method is also inadequate, however, as it does not use any of the information outside the null space. In [5], Yu et al. propose a direct LDA (DLDA) approach to solve this problem. It removes the null space of the between-class scatter matrix firstly by doing eigen-analysis. Then a simultaneous diagonalization procedure is used to seek the optimal discriminant vectors in the subspace of the between-class scatter matrix. However, in this method, removing the null space of the between-class scatter matrix by dimensionality reduction would indirectly lead to the losing of the null space of the within-class scatter matrix which contains considerable discriminative information. Rui Huang [10] proposed the method in which the null space of total scatter matrix which has been proved to be the common null space of both between-class and within-class scatter matrix, and useless for discrimination, is firstly removed. Then in the lowerdimensional projected space, the null space of the resulting within-class scatter matrix is calculated. This lower-dimensional null space, combined with the previous projection, represents a subspace of the whole null space of within-class scatter 
matrix, and is really useful for discrimination. The optimal discriminant vectors of LDA are derived from it.

In the LDA-based face recognition technique, the 2D face image matrices must be previously transformed into $1 \mathrm{D}$ image vectors. The resulting image vectors of faces usually lead to a high dimensional image vector space, where it is difficult to evaluate the between-class and within-class covariance matrices accurately due to its large size and the relatively small number of training samples. Fortunately, the eigenvectors can be calculated efficiently using the SVD techniques and the process of generating these covariance matrices is actually avoided. However, this does not imply that the eigenvectors can be evaluated accurately in this way since the eigenvectors are statistically determined by the between-class and within-class covariance matrices, no matter what method is adopted for obtaining them. In this paper, a new LDA approach called BLDA, is developed for image feature extraction. As opposed to conventional LDA, BLDA is based on 2D matrices rather than $1 \mathrm{D}$ vectors. That is, we firstly divides the original image into blocks. Then, we transform the image into a vector of blocks. By using row vector to represent each block, we can get the new matrix which is the representation of the image. Finally LDA can be applied directly on these matrices. In contrast to the between-class and within-class covariance matrices of LDA, the size of the image covariance matrix using BLDA is much smaller. As a result, BLDA has three important advantages over LDA. First, it is easier to evaluate the between-class and within-class covariance matrices accurately. Second, less time is required to determine the corresponding eigenvectors. And finally, block size could be changed to get the best results. The remainder of this paper is organized as follows: In Section 2, the traditional LDA method is reviewed. The idea of the proposed method and its algorithm are described in Section 3. In Section 4, experimental results are presented on the ORL face image database to demonstrate the effectiveness of our method. Finally, conclusions are presented in Section 5 .

\section{Linear Disciminant Analysis}

Let us consider a set of $N$ sample images $\left\{x_{1}, x_{2}, \ldots, x_{N}\right\}$ taking values in an $n$ dimensional image space, and assume that each image belongs to one of $C$ classes $\left\{C_{1}, C_{2}, \ldots, C_{c}\right\}$. Let $N_{i}$ be the number of the samples in class $C_{i}(i=1,2, \ldots, c)$, $\mu_{i}=\frac{1}{N_{i}} \sum_{x \in C_{i}} x$ be the mean of the samples in class $C_{i}, \mu=\frac{1}{N} \sum_{i=1}^{N} x_{i}$ be the mean of all samples. Then the between-class scatter matrix $S_{b}$ is defined as

$$
S_{b}=\frac{1}{N} \sum_{i=1}^{c} N_{i}\left(\mu_{i}-\mu\right)\left(\mu_{i}-\mu\right)^{T}=\frac{1}{N} \Phi_{b} \Phi_{b}^{T}
$$

and the within-class scatter matrix $S_{w}$ is defined as 


$$
S_{w}=\frac{1}{N} \sum_{i=1}^{c} \sum_{x_{k} \in C_{i}}\left(x_{k}-\mu_{i}\right)\left(x_{k}-\mu_{i}\right)^{T}=\frac{1}{N} \Phi_{w} \Phi_{w}^{T}
$$

Also, the total scatter matrix or mixture scatter matrix $S_{t}$ is defined as

$$
S_{t}=S_{b}+S_{w}=\frac{1}{N} \sum_{i=1}^{N}\left(x_{i}-\mu\right)\left(x_{i}-\mu\right)^{T}=\frac{1}{N} \Phi_{t} \Phi_{t}^{T}
$$

which is also the covariance matrix of all the samples.

In LDA, the projection $W_{\text {opt }}$ is chosen to maximize the ratio of the determinant of the between-class scatter matrix of the projected samples to the determinant of the within-class scatter matrix of the projected samples, i.e.,

$$
W_{\text {opt }}=\arg \max _{W} \frac{\left|W^{T} S_{b} W\right|}{\left|W^{T} S_{w} W\right|}=\left[w_{1} w_{2} \ldots w_{m}\right]
$$

where $\left\{w_{i} \mid i=1,2, \ldots, m\right\}$ is the set of generalized eigenvectors of $S_{b}$ and $S_{w}$ corresponding to the $m$ largest generalized eigenvalues $\left\{\lambda_{i} \mid i=1,2, \ldots, m\right\}$, i.e.,

$$
S_{b} w_{i}=\lambda_{i} S_{w} w_{i} \quad i=1,2, \ldots, m
$$

\section{Our proposed LDA}

In the LDA-based face recognition technique, the $2 \mathrm{D}$ face image matrices must be previously transformed directly into $1 \mathrm{D}$ image vectors. The resulting image vectors of faces usually lead to a high dimensional image vector space. However, in our proposed BLDA approach, we firstly divides the original image into $s=h x w$ size blocks with $h, w$ are the height and width of the block. Then, we transform the image into a vector of blocks. By using row vector $r$ with $r^{T} \in \mathbb{R}^{s}$ to represent each block, we can get the matrix $X \in \mathbb{R}^{k x s}$ which is the representation of the image, with $k$ is the number of blocks. See fig. 1 for the process.
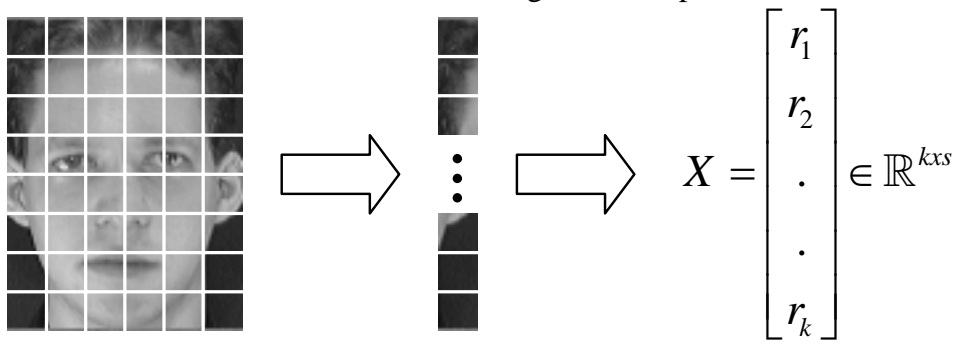
Fig. 1. The process of getting representation of each image

Now, set of $N$ sample images are represented as $\left\{X_{1}, X_{2}, \ldots, X_{N}\right\}$ with $X_{i} \in \mathbb{R}^{k x s}$. Then the between-class scatter matrix $S_{b}$ is re-defined as

$$
S_{b}=\frac{1}{N} \sum_{i=1}^{c} N_{i}\left(\mu_{C_{i}}-\mu_{X}\right)\left(\mu_{C_{i}}-\mu_{X}\right)^{T}
$$

and the within-class scatter matrix $S_{w}$ is re-defined as

$$
S_{w}=\frac{1}{N} \sum_{i=1}^{c} \sum_{X_{k} \in C_{i}}\left(X_{k}-\mu_{C_{i}}\right)\left(x_{k}-\mu_{C_{i}}\right)^{T}
$$

The total scatter matrix is re-defined as

$$
S_{T}=\frac{1}{N} \sum_{i=1}^{N}\left(X_{i}-\mu_{X}\right)\left(X_{i}-\mu_{X}\right)^{T}
$$

with $\mu_{X}=\sum_{i=1}^{N} X_{i} \in \mathbb{R}^{k x s}$ is the mean image of all samples and $\mu_{C_{i}}=\frac{1}{N_{i}} \sum_{X \in C_{i}} X$ be the mean of the samples in class $C_{i}$.

Similarly, a linear transformation mapping the original $k x s$ image space into an $m x s$ feature space, where $m<k$. The new feature matrices $Y_{i} \in \mathbb{R}^{m \times s}$ are defined by the following linear transformation :

$$
Y_{i}=W^{T}\left(X_{i}-\mu_{X}\right) \in \mathbb{R}^{m x s}
$$

where $i=1,2, \ldots, N$ and $W \in \mathbb{R}^{k x m}$ is a matrix with orthonormal columns. And the projection $W_{\text {opt }}$ is chosen with the criterion same as that in (4).

After a transformation by BLDA, a feature matrix is obtained for each image. Then, a nearest neighbor classifier is used for classification. Here, the distance between two arbitrary feature matrices $Y_{i}$ and $Y_{j}$ is defined by using Euclidean distance as follows :

$$
d\left(Y_{i}, Y_{j}\right)=\sqrt{\sum_{u=1}^{k} \sum_{v=1}^{s}\left(Y_{i}(u, v)-Y_{j}(u, v)\right)^{2}}
$$


Given a test sample $Y$, if $d\left(Y, Y_{c}\right)=\min _{j} d\left(Y, Y_{j}\right)$, then the resulting decision is $Y$ belongs to the same class as $Y_{c}$.

\section{Experimental results}

This section evaluates the performance of our propoped algorithm compared with that of the original LDA algorithm based on using ORL. In the ORL database, there are ten different images of each of 40 distinct subjects. For some subjects, the images were taken at different times, varying the lighting, facial expressions (open / closed eyes, smiling / not smiling) and facial details (glasses / no glasses). All the images were taken against a dark homogeneous background with the subjects in an upright, frontal position (with tolerance for some side movement).

In our experiments, firstly we tested the recognition rates with different number of training samples. $k(k=2,3,4,5)$ images of each subject are randomly selected from the database for training and the remaining images of each subject for testing. For each value of $k, 5$ runs are performed with different random partition between training set and testing set. The block size 3 by 3 is used in this first experiment, and two methods which are LDA and BLDA are performed. Table 1 . shows the recognition results of the best recognition accuracy among all the dimension of feature vectors. It means we test on all dimension of feature vectors and choose the best recognition accuracy.

Table 1. The recognition rates on ORL database with different training samples of two methods (LDA, BLDA - 3x3 block size)

\begin{tabular}{|c|c|c|c|c|}
\hline Training samples & 2 & 3 & 4 & 5 \\
\hline LDA (Fisherfaces) & 78.83 & 86.9 & 91.03 & 93.6 \\
\hline BLDA (3x3) & 86.22 & 89.61 & 93.53 & 95.83 \\
\hline
\end{tabular}

Next, we try to test BLDA approach performance when the block size is changed. And several results can be show in the Table 2. The same protocol as previous experiments, we choose the recognition result of the dimension feature vectors which give the best accuracy.

Table 2. The recognition rates with different block sizes.

\begin{tabular}{|c|c|c|c|c|}
\hline & \multicolumn{4}{|c|}{ Training samples } \\
\hline Size of block & 2 & 3 & 4 & 5 \\
\hline$[2 \times 2]$ & 86.77 & 90.4 & 94.23 & 96.48 \\
\hline$[3 \times 3]$ & 86.22 & 89.61 & 93.53 & 95.83 \\
\hline$[5 \times 5]$ & 87.88 & 90.92 & 94.86 & 96.89 \\
\hline$[10 \times 2]$ & 86.17 & 90.41 & 93.6 & 95.98 \\
\hline$[10 \times 10]$ & 83.51 & 88.52 & 90.55 & 93.57 \\
\hline
\end{tabular}


From Table 2, it seems to be that the block size $5 \times 5$ give the best recognition results among all. However we still not yet find the relationship between the block size and the recognition result.

\section{Conclusions}

A new LDA-based method (BLDA) for face recognition has been proposed in this paper. As opposed to conventional LDA, BLDA is based on 2D matrices rather than $1 \mathrm{D}$ vectors. That is, we firstly divides the original image into blocks. Then, we transform the image into a vector of blocks. By using row vector to represent each block, we can get the new matrix which is the representation of the image. Finally LDA can be applied directly on these matrices. In contrast to the covariance matrix of LDA, the size of the image covariance matrix using BLDA is much smaller. As a result, BLDA has three important advantages over LDA. First, it is easier to evaluate the covariance matrix accurately. Second, less time is required to determine the corresponding eigenvectors. And finally, block size could be changed to get the best results.

\section{Reference}

1. W. Zhao, R. Chellappa, A. Rosenfeld, J. Phillips: Face Recognition: A Literature Survey. Technical Report, CFAR-TR00-948, Univ. of Maryland, 2000. (Revised 2002)

2. M Turk, A Pentland: Eigenfaces for recognition. Journal of Cognitive Neuroscience, vol. 3 (1991) 71-86.

3. Jian Yang, Zhang D., Frangi A.F., Jing-yu Yang: Two-dimensional PCA: a new approach to appearance-based face representation and recognition. Pattern Analysis and Machine Intelligence, IEEE Transactions on , vol. 26 (2004) 131 - 137.

4. P. N. Belhumeur, J. P. Hespanha, D. J. Kriegman: Eigenfaces vs. fisherface: Recognition using class specific linear projection. IEEE Trans. Pattern Anal. Machine Intel.., vol. 19 (1997) 711-720.

5. H. Yu, J. Yang: A direct LDA algorithm for high-dimensional data with application to face recognition. Pattern Recognit., vol. 34 (2001) 2067-2070.

6. M. Loog, R. P. W. Duin, R. Haeb-Umbach: Multiclass linear dimension reduction by weighted pairwise fisher criteria. IEEE Trans. Pattern Anal. Machine Intell., vol. 23 (2001) 762-766.

7. A. M. Martinez, A. C. Kak: PCA versus LDA. IEEE Trans. Pattern Anal. Machine Intell., vol. 23 (2001) 228-233.

8. D. H. Foley, J. W. Sammon: An optimal set of discrimination vectors. IEEE Trans. Comput., vol. C-24 (1975) 281-289.

9. R. A. Fisher: The use of multiple measurements in taxonomic problems. Ann. Eugenics, vol. 7 (1936) 178-188.

10. Rui Huang, Qingshan Liu, Hanqing Lu, Songde Ma: Solving the small sample size problem of LDA. Proceedings. 16th International Conference on Pattern Recognition, vol. 3 (2002). 
Vo Dinh Minh Nhat, and Sungyoung Lee

11. C. Liu, H. Wechsler: Robust coding scheme for indexing and retrieval from large face databases. IEEE Trans. Image Processing, vol. 9 (2000) 132-137.

12. Chengjun Liu, Wechsler H.: A shape- and texture-based enhanced Fisher classifier for face recognition. IEEE Trans. Image Processing, vol. 10 (2001) 598-608.

13. L. Chen, H. M. Liao, M. Ko, J. Lin, G. Yu: A new LDA-based face recognition system which can solve the small sample size problem. Pattern Recognit., vol. 33 (2000) 17131726. 